\title{
CircRNA_0044556 diminishes the sensitivity of triple-negative breast cancer cells to adriamycin by sponging miR-145 and regulating NRAS
}

\author{
JINGJING CHEN ${ }^{1,2 *}$, PENG SHI ${ }^{3 *}$, JINGHUA ZHANG $^{4}$, YUFENG LI $^{5}$, \\ $\mathrm{JIE} \mathrm{MA}^{2}$, YUDONG $\mathrm{ZHANG}^{6}$ and HONG LIU ${ }^{1}$
}

${ }^{1}$ The Second Department of Breast Cancer, Tianjin Medical University Cancer Institute and Hospital, Tianjin Medical University, National Clinical Research Center for Cancer, Tianjin's Clinical Research Center for Cancer, Key Laboratory of Cancer Prevention and Therapy, Key Laboratory of Breast Cancer Prevention and Therapy, Tianjin 300060;

${ }^{2}$ The First Department of Breast Surgery; ${ }^{3}$ The Second Department of Urology Surgery, Tangshan People's Hospital;

${ }^{4}$ Department of Breast Health Care, Maternal and Child Care Service Centre; ${ }^{5}$ Central Laboratory,

Tangshan People's Hospital, Tangshan, Hebei 063000; ${ }^{6}$ North China University of Science and Technology,

Tangshan People's Hospital, Tangshan, Hebei 063210, P.R. China

Received August 12, 2021; Accepted November 22, 2021

DOI: $10.3892 / \mathrm{mmr} .2021 .12567$

\begin{abstract}
CircRNAs are associated with adriamycin (ADM) resistance in triple-negative breast cancer (TNBC), but the mechanism is unknown. Reverse transcription-quantitative PCR was applied to quantify circular RNA (circRNA)_0044556, microRNA (miR)-145 and NRAS proto-oncogene, GTPase (NRAS) in TNBC tissues and cells with or without ADM treatment. Following ADM treatment, the effects of circRNA_0044556 on the viability, ADM resistance, apoptosis and migration of TNBC cells were investigated by cell function experiments (Cell Counting Kit-8, flow cytometry and Transwell assays). The targeting relationship between circRNA_0044556 and miR-145 was investigated via bioinformatics analysis, dual-luciferase reporter assay and RNA immunoprecipitation. The effects of the circRNA_0044556/miR-145 axis on the TNBC cells were revealed by rescue experiments. Correlations among circRNA_0044556, miR-145 and NRAS were analyzed by Pearson's correlation test. CircRNA_0044556 was
\end{abstract}

Correspondence to: Dr Hong Liu, The Second Department of Breast Cancer, Tianjin Medical University Cancer Institute and Hospital, Tianjin Medical University, National Clinical Research Center for Cancer, Tianjin's Clinical Research Center for Cancer, Key Laboratory of Cancer Prevention and Therapy, Key Laboratory of Breast Cancer Prevention and Therapy, 1 Huanhu West Road and Tiyuan North Road, Tianjin 300060, P.R. China

E-mail: liuhon_lliho@163.com

*Contributed equally

Key words: triple-negative breast cancer, adriamycin, chemotherapy sensitivity, circular RNA_0044556, microRNA-145 highly expressed in TNBC tissues and cells with or without ADM-resistance. The overexpression of circRNA_0044556 promoted cell viability, ADM-resistance and migration, while inhibiting the apoptosis by sponging miR-145. Upregulation of miR-145 reversed the effects of circRNA_0044556 on the TNBC cells. CircRNA_0044556 was negatively correlated with miR-145 yet positively correlated with NRAS, the target gene of miR-145, in addition to the discovery suggesting the negative regulatory effects of circRNA_0044556 on miR-145. CircRNA_0044556 diminished the sensitivity of TNBC cells to ADM via the miR-145/NRAS axis.

\section{Introduction}

Breast cancer is a malignant tumor that has a variety of clinicopathological features due to its heterogeneous nature (1), which is mainly classified on the basis of the specific immunohistochemical indicators as luminal breast cancer, human epidermal growth factor receptor 2 (HER-2)-overexpressed breast cancer and triple-negative breast cancer (TNBC) (2). TNBC patients are diagnosed with negative indicators of estrogen receptor (ER), progesterone receptor (PR) and HER-2, which account for $\sim 10-24 \%$ of all cases with breast cancer (3). Compared with other subtypes, TNBC is associated with the worst curative outcome and the highest mortality owing to its biological characteristics of strong invasion, high risk of early recurrence and rapid rate of distant metastasis $(4,5)$. Therefore, improving the efficacy of the treatment of TNBC is currently one of the greatest challenges in the research field of breast carcinoma.

Adriamycin (ADM) belongs to anthracyclines and has a very broad antitumor spectrum and is commonly used in the chemotherapy regimen for breast cancer (6). ADM acts as a suppressor of the growth of tumor cells mainly by blocking the synthesis of nucleic acids, for instance (7). However, a high 
rate of resistance to ADM in clinical chemotherapy for TNBC has been reported, but there is a lack of effective therapeutic measures (8). Based on this discovery, it is of great significance to explore the mechanism underlying the resistance to ADM in TNBC cells so as to improve the chemotherapeutic efficacy.

Circular RNA (circRNA) is a new type of RNA different from linear RNA and is more stable in expression due to its closed-loop molecular structure, which makes it less susceptible to Ribonuclease R (RNase R) (9). It has been shown in previous studies that circRNAs are widely present in the human body and have biological functions, such as the participation as competing endogenous RNAs (ceRNAs), the regulation of variable shearing and transcription, and the translation of protein, in addition to the control of normal physiological activities and the development of tumors $(10,11)$. At present, increasing evidence suggests that circRNAs are closely engaged in the proliferation, invasion, apoptosis and drug resistance of multiple tumor cells, including breast cancer cells, and are expected to be the therapeutic targets or prognostic markers for cancers (12-14). In a study regarding the aberrant expression profile of circRNAs in drug-resistant breast cancer cells, circRNA_0044556 was revealed to be upregulated (6), but the detailed mechanism remains to be further elucidated.

In the present study, following the quantification of the expression of circRNA_0044556 in TNBC, its role in regulating the sensitivity of TNBC cells to ADM was investigated and its related molecular mechanism was revealed by cell functional experiments, aiming to provide a rationale for the selection of circRNAs as molecular targets in improving the sensitivity of TNBC to chemotherapy.

\section{Materials and methods}

Ethical statement and sample collection. TNBC tissues and the corresponding adjacent normal tissues were collected from 40 patients (aged $26-58$ years) with TNBC who underwent tumor resection at Tangshan People's Hospital (Tangshan, China) between April 2019 and July 2020, upon obtaining written informed consents from the donors. ADM-resistant tissue samples $(\mathrm{n}=15)$ and ADM-sensitive tissue samples $(n=25)$ were acquired from patients who met the Response Evaluation Criteria in Solid Tumors (15). The present study was approved (approval no. TNBC20190304) by the Ethics Committee of Tangshan People's Hospital (Tangshan, China).

Cell culture. Normal mammary epithelial cell line MCF-10A (CRL-10317), and TNBC cell lines MDA-MB-231 (CRM-HTB-26), MDA-MB-453 (HTB-131), MDA-MB-157 (HTB-24) and BT549 (HTB-122) were obtained from American Type Culture Collection (ATCC). ADM-resistant cell line MDA-MB-231/ADM (IMD-003) was purchased from Xiamen Immocell Biotechnology Co., Ltd. DMEM (cat. no. SNM-002C; Sunncell) supplemented with 10\% fetal bovine serum (FBS; cat. no. SNS-002; Sunncell) was used for the culture of all cells at $37^{\circ} \mathrm{C}$ with $5 \% \mathrm{CO}_{2}$ as previously described (6). Subsequently, reverse transcription-quantitative polymerase chain reaction (RT-qPCR) was carried out to quantify the expression of circRNA_0044556 in these cells.

Cell transfection and grouping. To determine the role circRNA_0044556 plays in TNBC, MDA-MB-231 and MDA-MB-231/ADM cells were subjected to the transfection. CircRNA_0044556-overexpression plasmid was synthesized and obtained from BersinBio, the empty plasmid as the control, and then these plasmids were transfected at $37^{\circ} \mathrm{C}$ and for $48 \mathrm{~h}$ into the MDA-MB-231 cells which were used to establish the groups of circRNA_0044556 and negative control (NC), respectively, while the cells without any transfection or treatment served as the control. Likewise, small interfering (si)RNA against circRNA_0044556 (50 pmol, si-circRNA_0044556, 5'-AGCCACAAAGAGTCTACATGTCT-3') and its negative control (50 pmol, si-NC, 5'-UUCUCCGAACGUGUCAC GU-3'), purchased from Shanghai GenePharma Co., Ltd., were independently transfected into MDA-MB-231/ADM cells, and the Control group was established as well. In the further investigation on the molecular interplay, $100 \mathrm{nM}$ microRNA (miR)-145 mimic (M, miR10000437-1-5, 5'-GUCCAGUUU UCCCAGGAAUCCCU-3'; Guangzhou RiboBio Co., Ltd.) or $100 \mathrm{nM}$ mimic control (MC, 5'-UUCUUCGAACGUGUC ACGUTT-3'; Guangzhou RiboBio Co., Ltd.) was transfected into the parental MDA-MB-231 cells, and $100 \mathrm{nM}$ miR-145 inhibitor (I, miR20000437-1-5, 5'-AGGGAUUCCUGGGAA AACUGGAC-3'; Guangzhou RiboBio Co., Ltd.) or $100 \mathrm{nM}$ inhibitor control (IC, 5'-CAGUACUUUUGUGUAGUA CAA-3') was transfected into MDA-MB-231/ADM cells at $37^{\circ} \mathrm{C}$ for $48 \mathrm{~h}$. All transfections on the cells were carried out using Lipofectamine ${ }^{\circledR} 2000$ (cat. no. 11668019; Invitrogen; Thermo Fisher Scientific, Inc.), and the transfected cells were assigned to the groups as follows: circRNA_0044556 group, si-circRNA_0044556 group, NC group, si-NC group, circRNA_0044556+M group, si-circRNA_0044556+I group, circRNA_0044556+MC group, si-circRNA_0044556+IC group, si-NC+I group, $\mathrm{NC}+\mathrm{M}$ group, $\mathrm{NC}+\mathrm{MC}$ group and si-NC+IC group. At $48 \mathrm{~h}$ after transfection, cells were collected for subsequent experiments.

Total RNA isolation and RT-qPCR. Total RNA was separated from tissue samples and cells by TRIzol reagent (cat. no. 15596026; Invitrogen; Thermo Fisher Scientific, Inc.). Then, the extracted RNA was reversely transcribed into complementary DNA (cDNA) using a reverse transcription kit (cat. no. 18090010; Invitrogen; Thermo Fisher Scientific, Inc.) according to the manufacturer's protocols. Subsequently, the expression levels of circRNA_0044556, miR-145 and NRAS proto-oncogene, GTPase (NRAS) were evaluated by RT-qPCR using qPCR SYBR Green Master Mix (cat. no. Q121-02; Vazyme Biotech Co., Ltd.) on an ABI 7500 system (Applied Biosystems; Thermo Fisher Scientific, Inc.). The thermocycling conditions were set as follows: Predenaturation at $95^{\circ} \mathrm{C}$ for $5 \mathrm{~min}, 40$ cycles of $95^{\circ} \mathrm{C}$ for $10 \mathrm{sec}$ and $60^{\circ} \mathrm{C}$ for $35 \mathrm{sec}$. Glyceraldehyde-3-phosphate dehydrogenase (GAPDH) and U6 were used for the normalization of the expression levels of circRNA_0044556, NRAS, and miR-145. The expression levels of circRNA_0044556/NRAS (relative to GAPDH) and miR-145 (relative to U6) were calculated by the $2^{-\Delta \Delta C q}$ method (16). Primer sequences used in this experiment were 
as follows: circRNA_0044556 forward, 5'-TGACGAGAC CAAGAACTGCC-3' and reverse, 5'-GCACCATCATTTCCA CGAGC-3'; miR-145 forward, 5'-CAGTCTTGTCCAGTTTTC CCAG-3' and reverse, 5'-TATGCTTGTTCTCGTCTCTGT GTC-3'; NRAS forward, 5'-ATGACTGAGTACAAACTG GTGGT-3' and reverse, 5'-CATGTATTGGTCTCTCATGGC AC-3'; U6 forward, 5'-ATTGGAACGATACAGAGAAGA TT-3' and reverse, 5'-GGAACGCTTCACGAATTTG-3'; and GAPDH forward, 5'-GAAGGTGAAGGTCGGAGTC-3' and reverse, 5'-GAAGATGGTGATGGGATTTC-3'.

Cell viability evaluation. To explore the role of circRNA_0044556 in the viability of TNBC cells to ADM, Cell Counting Kit-8 (CCK-8) assay was applied. Firstly, parental and ADM-resistant MDA-MB-231 cells $\left(2 \times 10^{4}\right)$ were seeded into a 96-well plate and treated with different concentrations of $\operatorname{ADM}(0,0.1,0.2,0.4,0.8,1.6,3$ and $6 \mu \mathrm{g} / \mathrm{ml})$ at $37^{\circ} \mathrm{C}$ for $24 \mathrm{~h}$, followed by the addition of $10 \mu \mathrm{l} \mathrm{CCK}-8$ solution (cat. no. CK04; Dojindo Molecular Technologies, Inc.) to treat cells in each well at $37^{\circ} \mathrm{C}$ with $5 \% \mathrm{CO}_{2}$ for $2 \mathrm{~h}$. A microplate reader (Infinite M200; Tecan Group, Ltd.) was used to measure the optical density (OD) value at a wavelength of $490 \mathrm{~nm}$, and then the half maximal inhibitory concentration $\left(\mathrm{IC}_{50}\right)$ value was calculated.

Cell apoptosis analysis. Annexin V/FITC apoptosis detection kit (cat. no. AD10; Dojindo Molecular Technologies, Inc.) was used for assessing the apoptotic capacity of TNBC cells undergoing the indicated treatments (parental and ADM-resistant MDA-MB-231 cells transfected with circRNA_0044556, si-circRNA_0044556, miR-145 mimic, inhibitor and their negative control). According to the description in the manual, cell suspension $\left(1 \times 10^{6}\right)$ was prepared using 1X Annexin V Binding Solution, and was then incubated with $5 \mu \mathrm{l}$ Annexin V/FITC solution and $5 \mu 1$ PI solution at room temperature for $15 \mathrm{~min}$ in the dark. Next, the stained samples were analyzed using an Accuri C6 flow cytometer with CFlow software (v. 1.32; BD Biosciences) to determine the apoptosis.

Cell migration detection. Transwell inserts $(8 \mu \mathrm{m}$; product number 351184; Corning, Inc.) were used to conduct the Transwell assay. Following transfection, parental and ADM-resistant MDA-MB-231 cells were resuspended at a density of $4 \times 10^{4}$ cells/well with $5 \mu 1$ serum-free medium in the upper chamber of the insert, whereas $500 \mu \mathrm{l}$ culture medium containing $10 \%$ FBS was added in the lower chamber at the same time. After $48 \mathrm{~h}$ of incubation at $37^{\circ} \mathrm{C}$, the residual cells on the upper chamber were removed with a cotton swab, and the membrane was subjected to fixation with $4 \%$ paraformaldehyde (cat. no. E672002; Sangon Biotech Co., Ltd.) at $4^{\circ} \mathrm{C}$ for $30 \mathrm{~min}$ and staining was performed using $0.1 \%$ crystal violet for $15 \mathrm{~min}$ at room temperature (cat. no. G1064; Beijing Solarbio Science and Technology Co., Ltd.). Finally, a light microscope (x250, magnification; CX23; Olympus Corporation) was used to observe the migration of cells on the lower side of the membrane.

Bioinformatics analysis and dual-luciferase reporter assay. The targeting relationship among circRNA_0044556, miR-145, and NRAS in TNBC cells was predicted by circInteractome (https://circinteractome.irp.nia.nih.gov/) and StarBase v2.0 (http://starbase.sysu.edu.cn/index.php), respectively.

For verifying the targeting relationship through dual luciferase reporter assay, vectors (cat. no. E1330; Promega Corporation) were used to construct wild-type (wt) reporter plasmid of circRNA_0044556 (circRNA_0044556-wt; 5'-GGUGCCAAGGGUCUGACUGGAAG-3'), mutant (mut) plasmid of circRNA_0044556 (circRNA_0044556-mut; 5'-GGUGCCAAGGGUCUGCCUCGUAG-3'), NRAS-wt reporter plasmid (5'-CAAACCCUUUACCAUGACUGG AA-3') and NRAS-mut reporter plasmid (5'-CAAACCCUU UACCCUGUCUCGUA-3'). Next, MDA-MB-231 cells were co-transfected with miR-145 mimic (5'-GUCCAGUUU UCCCAGGAAUCCCU-3') or its miR-NC (5'-UUCUUC GAACGUGUCACGUTT-3') and the reporter plasmids (circRNA_0044556-wt, circRNA_0044556-mut, NRAS-wt or NRAS-mut) using Lipofectamine ${ }^{\circledR} 2000$ reagent (Invitrogen; Thermo Fisher Scientific, Inc.). After 48 h, the relative luciferase activity was determined by a Dual Luciferase Reporter Gene Assay Kit (cat. no. 11402ES60; Shanghai Yeasen Biotechnology Co., Ltd.). The luciferase activity was normalized to the Renilla luciferase activity.

RNA binding protein immunoprecipitation (RIP). RIP kit (cat. no. KT102-01), obtained from Guangzhou Saicheng Biotechnology Co., Ltd. was used to determine the connectivity between circRNA_0044556 and miR-145. Briefly, the transfected cells $\left(4 \times 10^{7}\right.$ cells) were collected with phosphate-buffered saline (cat. no. C0221A; Beyotime Institute of Biotechnology) and lysed with cell lysis buffer. G/A beads $(100 \mu \mathrm{l})$ were prepared and divided into the groups argonaute2 (Ago2) and immunoglobulin $\mathrm{G}(\operatorname{IgG})$ (4). Then, the antibodies against Ago2 (product code ab32381, 1:1,000) and IgG (product code ab133470, 1:1,000; both from Abcam) were added into the lysates of cells in the indicated groups at $4^{\circ} \mathrm{C}$. After $6 \mathrm{~h}$ of incubation, the antibody-tagged beads were incubated with the lysates at $4^{\circ} \mathrm{C}$ overnight. Subsequently, the beads were washed with RIP buffer for five times by centrifugation at $1,500 \mathrm{x} \mathrm{g}$ for $2 \mathrm{~min}$ at $4^{\circ} \mathrm{C}$. Proteinase $\mathrm{K}$ was added and incubated for $45 \mathrm{~min}$ at $65^{\circ} \mathrm{C}$ to remove the protein prior to RNA isolation, and RNA was isolated using TRIzol ${ }^{\circledR}$ reagent (Thermo Fisher Scientific, Inc.) as aforementioned. RNA was purified and precipitation according to the manufacturer's protocols. The immunoprecipitated RNA was subjected to RT-qPCR to determine the enrichment of circRNA_0044556 in MDA-MB-231 cells with miR-145 overexpression.

Statistical analysis. All data were analyzed using GraphPad Prism 8.0 software (GraphPad Software, Inc.), and measurement data were expressed as the mean \pm standard deviation. The differences between two independent samples or two paired samples were analyzed by independent samples $t$-test or paired samples t-test. One-way analysis of variance with Tukey's post hoc test was applied for single-factor differences between multiple groups, while two-way analysis of variance for the two-factor differences between multiple groups. The correlation among circRNA_0044556, miR-145 and NRAS in TNBC was analyzed with Pearson's correlation test. $\mathrm{P}<0.05$ was considered to indicate a statistically significant difference. 

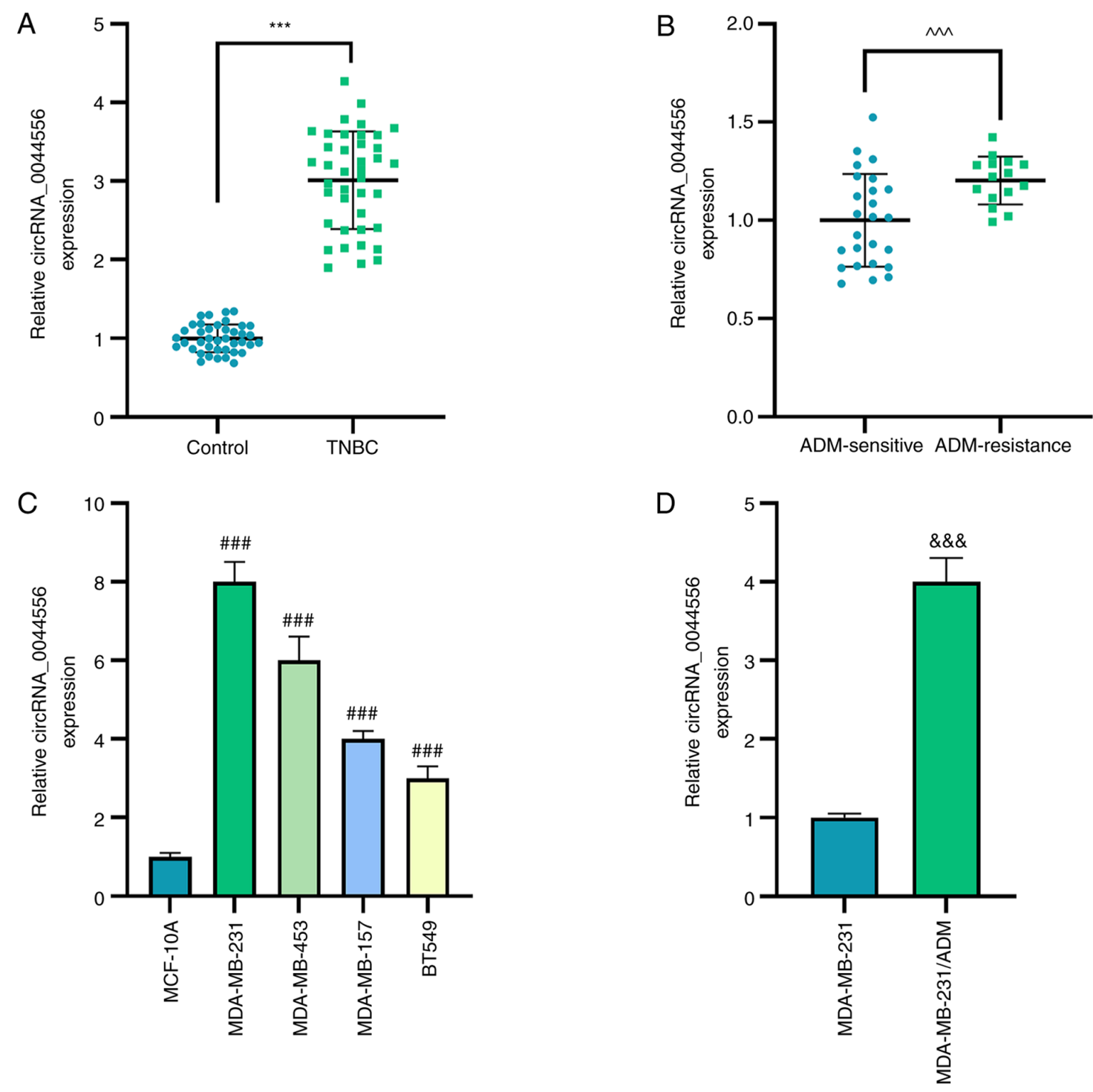

Figure 1. Expression of circRNA_0044556 in TNBC tissues and TNBC cells with or without ADM treatment. (A-D) The expression of circRNA_0044556 was quantified in normal mammary epithelial cells, TNBC tissues and TNBC cells with or without ADM-resistance by reverse transcription-quantitative PCR.

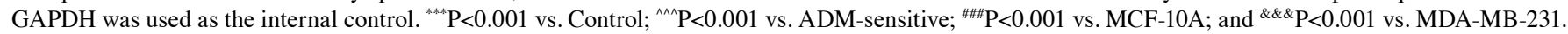
circRNA, circular RNA; TNBC, triple-negative breast cancer; ADM, adriamycin.

\section{Results}

Quantification of circRNA_0044556 expression in TNBC tissues and cells with or without ADM treatment. RT-qPCR was first performed to quantify the expression of circRNA_0044556 in tissues (TNBC tissues with or without ADM resistance and the adjacent tissue) and cells (mammary epithelial cell line MCF-10A, TNBC cell lines and MDA-MB-231/ADM cells). As revealed in Fig. 1A-D, circRNA_0044556 was highly expressed in TNBC tissues, ADM-resistant TNBC tissues and TNBC cells $(\mathrm{P}<0.001)$. As the most significantly high expression of circRNA_0044556 was detected in MDA-MB-231 cells among the four other TNBC cell lines, MDA-MB-231 cells were selected for subsequent experiments.

CircRNA_0044556 regulates the viability of ADM-treated $M D A-M B-231$ and $M D A-M B-231 / A D M$ cells. Subsequently, MDA-MB-231 cells were transfected with circRNA_0044556 overexpression plasmid, and MDA-MB-231/ADM cells were transfected with si-circRNA_0044556. RT-qPCR was used to estimate the transfection efficiency and it was determined that the expression of circRNA_0044556 was significantly promoted by the overexpression plasmid of circRNA_0044556, yet it was suppressed by si-circRNA_0044556 (Fig. 2A and B; $\mathrm{P}<0.001)$. It was indicated in the results of the CCK-8 assay that after the treatment of ADM in gradient concentrations, overexpressed circRNA_0044556 enhanced the viability of parental MDA-MB-231 cells and increased the $\mathrm{IC}_{50}$ value (Fig. $2 \mathrm{C}$ and D; $\mathrm{P}<0.05)$, whereas the silencing of circRNA_0044556 significantly reduced the viability of MDA-MB-231/ADM cells and decreased the $\mathrm{IC}_{50}$ value (Fig. $2 \mathrm{E}$ and $\mathrm{F} ; \mathrm{P}<0.01$ ).

CircRNA_0044556 affects the apoptotic and migratory capacities of TNBC cells with or without ADM treatment. It was demonstrated in the apoptotic assay (Fig. 3A and B) that the overexpression of circRNA_0044556 decreased the percentage of apoptotic parental TNBC cells $(\mathrm{P}<0.001)$, whereas the silencing of circRNA_0044556 promoted apoptosis of the ADM-resistant 
A

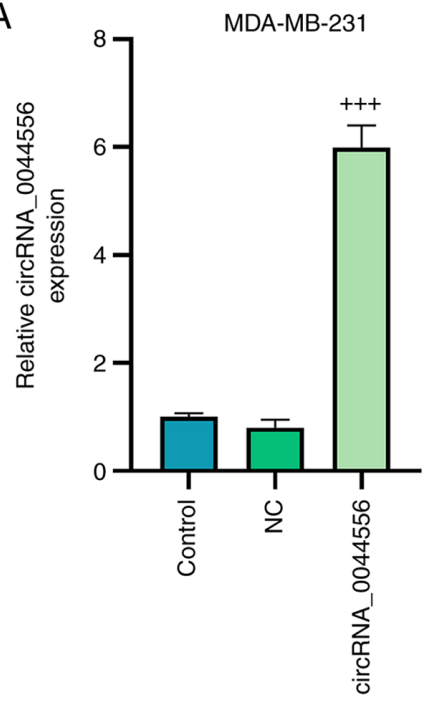

C

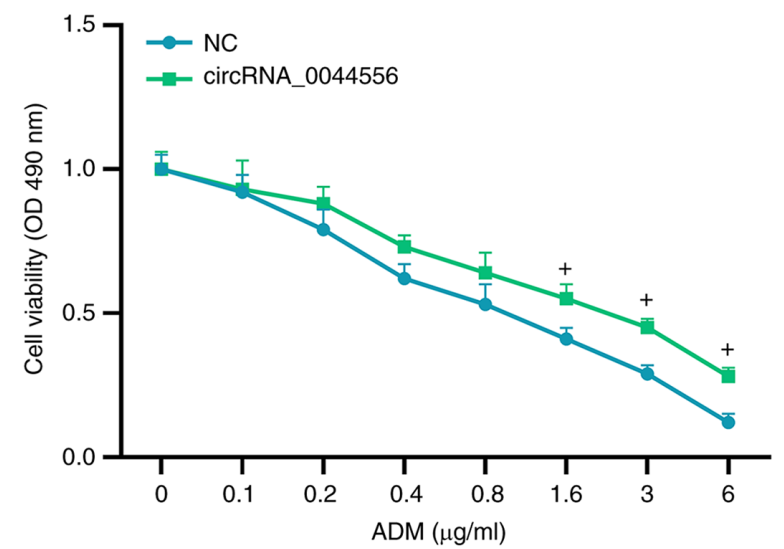

E

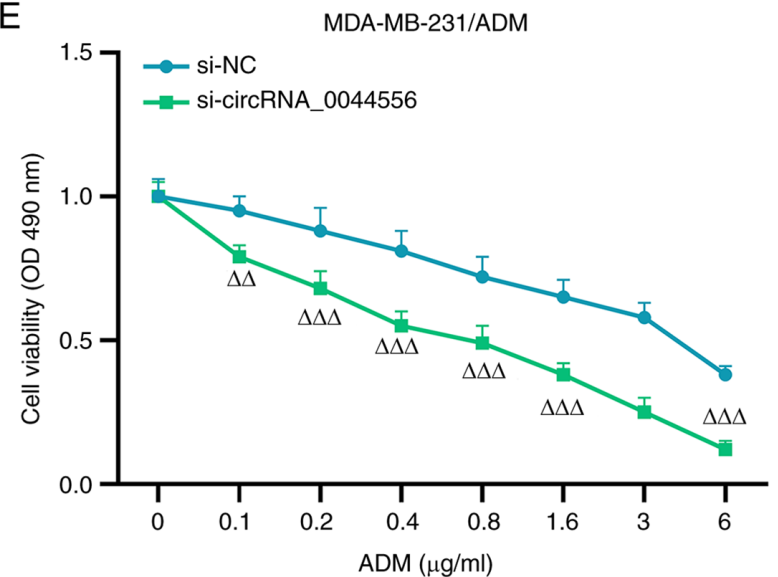

B

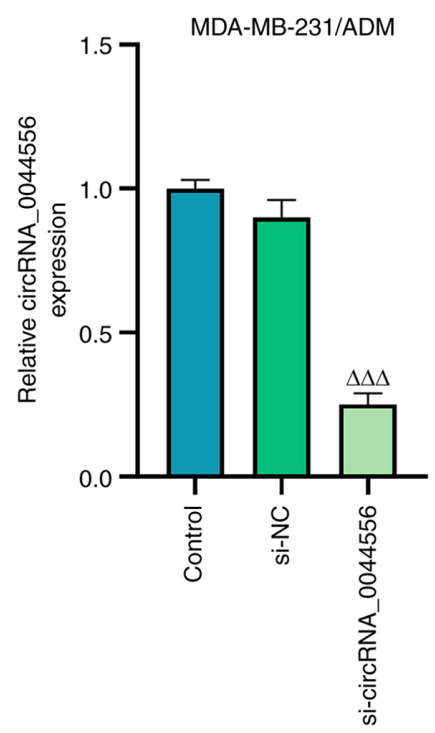

D

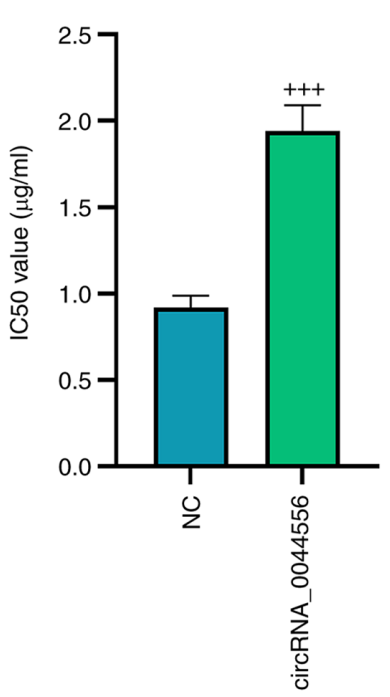

$\mathrm{F}$

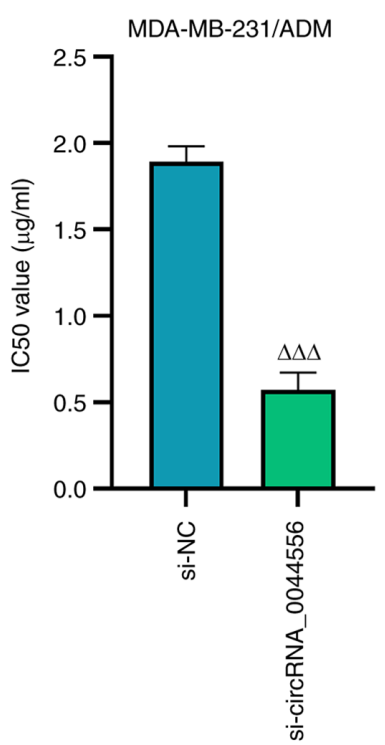

Figure 2. CircRNA_0044556 plays a role in the viability of TNBC cells with or without ADM treatment. (A and B) Reverse transcription-quantitative PCR was performed to evaluate the expression of circRNA_0044556 following the overexpression or silencing of circRNA_0044556 in MDA-MB-231 cells with or without ADM treatment. GAPDH was used as the internal control. (C and D) A CCK-8 assay was used to determine the viability of parental MDA-MB-231 cells after gradient treatments of $\mathrm{ADM}$, and the $\mathrm{IC}_{50}$ value was calculated. (E and F) A CCK-8 assay was used to evaluate the viability of MDA-MB-231/ADM cells after gradient treatments of $\mathrm{ADM}$, and the $\mathrm{IC}_{50}$ value was calculated. ${ }^{+} \mathrm{P}<0.05$ and ${ }^{+++} \mathrm{P}<0.001 \mathrm{vs}$. $\mathrm{NC} ;{ }^{\Delta \Delta} \mathrm{P}<0.01$ and ${ }^{\Delta \Delta \Delta} \mathrm{P}<0.001 \mathrm{vs}$. si-NC. circRNA, circular RNA; TNBC, triple-negative breast cancer; ADM, adriamycin; CCK-8, Cell Counting Kit-8; $\mathrm{IC}_{50}$, half maximal inhibitory concentration; si-, small interfering; $\mathrm{NC}$, negative control. 

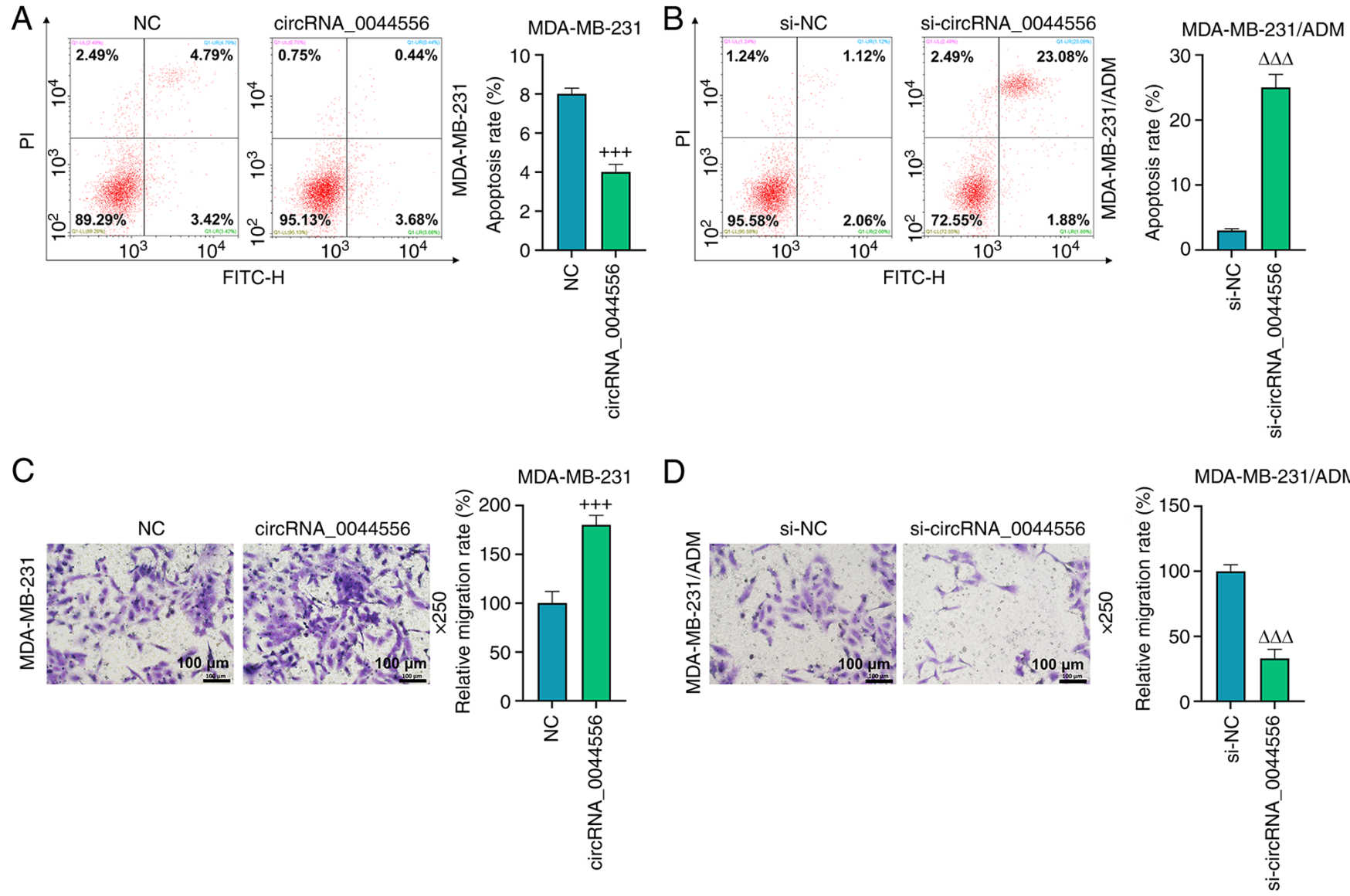

Figure 3. CircRNA_0044556 plays a role in the apoptosis and migration of TNBC cells with or without ADM treatment. (A and B) Flow cytometry was employed to analyze the apoptosis following the overexpression or silencing of circRNA_0044556 in MDA-MB-231 cells with or without ADM treatment. (C and D) Transwell assay was performed to detect the migration capacity of transfected cells. ${ }^{+++} \mathrm{P}<0.001 \mathrm{vs}$. NC; and ${ }^{\Delta \Delta \Delta} \mathrm{P}<0.001 \mathrm{vs}$. si-NC. circRNA, circular RNA; TNBC, triple-negative breast cancer; ADM, adriamycin; si-, small interfering; NC, negative control.

cells $(\mathrm{P}<0.001)$. As demonstrated in Fig. $3 \mathrm{C}$ and $\mathrm{D}$, a distinctly increased number of migratory TNBC cells were observed after the overexpression of circRNA_0044556 compared with those in the $\mathrm{NC}$ group $(\mathrm{P}<0.001)$, whereas the migratory capacity of MDA-MB-231/ADM cells was significantly suppressed after the inhibition of circRNA_0044556 as compared with the si-NC group $(\mathrm{P}<0.001)$.

CircRNA_0044556 sponges miR-145, which is expressed at a low level in TNBC tissues and TNBC cells with or without ADM treatment. The binding site between the 3'-untranslated region (UTR) of circRNA_0044556 and the seed region of miR-145 (Fig. 4A) was predicted by bioinformatics analysis. Subsequently, the dual-luciferase reporter assay in Fig. 4B indicated that miR-145 mimic significantly suppressed the luciferase activity of circRNA_0044556-wt $(\mathrm{P}<0.001)$. The binding between circRNA_0044556 and miR-145 in TNBC cells was further determined via RIP assay, and it was revealed that circRNA_0044556 was enriched in Ago2-tagged beads compared with the control group of IgG (Fig. 4C; $\mathrm{P}<0.001$ ). Notably, detection with RT-qPCR revealed that the expression of miR-145 was downregulated in TNBC tissues, and ADM-resistant TNBC tissues in particular (Fig. 4D and E; $\mathrm{P}<0.001)$. Additionally, according to Pearson's correlation analysis (Fig. 4F), miR-145 was negatively correlated with
circRNA_0044556 in TNBC patients with ADM resistance $(\mathrm{r}=-0.6168, \mathrm{P}=0.0143)$. Correspondingly, a lower expression of miR-145 in TNBC cell lines and MDA-MB-231/ADM cells was also identified (Fig. 4G and $\mathrm{H}$; $\mathrm{P}<0.001$ ).

Overexpressed circRNA_0044556 attenuates apoptosis and enhances migration of TNBC cells with or without ADM treatment by targeting miR-145. In order to understand the interplay between circRNA_0044556 and miR-145 in TNBC, miR-145 mimic and circRNA_0044556-overexpressing plasmid or miR-145 inhibitor and si-circRNA_0044556 were co-transfected in parental and ADM-resistant MDA-MB-231 cells, and the transfection efficiency was evaluated by RT-qPCR. As revealed in Fig. 5A, the overexpression of circRNA_0044556 downregulated miR-145 expression and miR-145 mimic induced upregulation of miR-145 compared with the $\mathrm{NC}+\mathrm{MC}$ group, the trends of which were both overturned in the circRNA_0044556+M group $(\mathrm{P}<0.001)$. Conversely, si-circRNA_0044556 upregulated miR-145 expression and miR-145 inhibitor led to decreased miR-145 expression compared with the si- $\mathrm{NC}+\mathrm{IC}$ group, and these trends were both reversed in the si-circRNA_0044556+I group (Fig. 5B; $\mathrm{P}<0.01$ ). Subsequently, the results of rescue experiments indicated that the high expression of miR-145 induced the apoptosis, decreased the migration of parental 
A

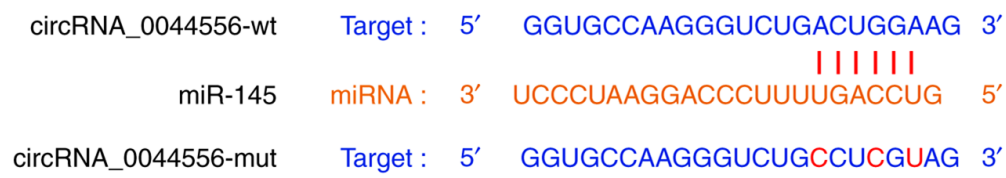

C
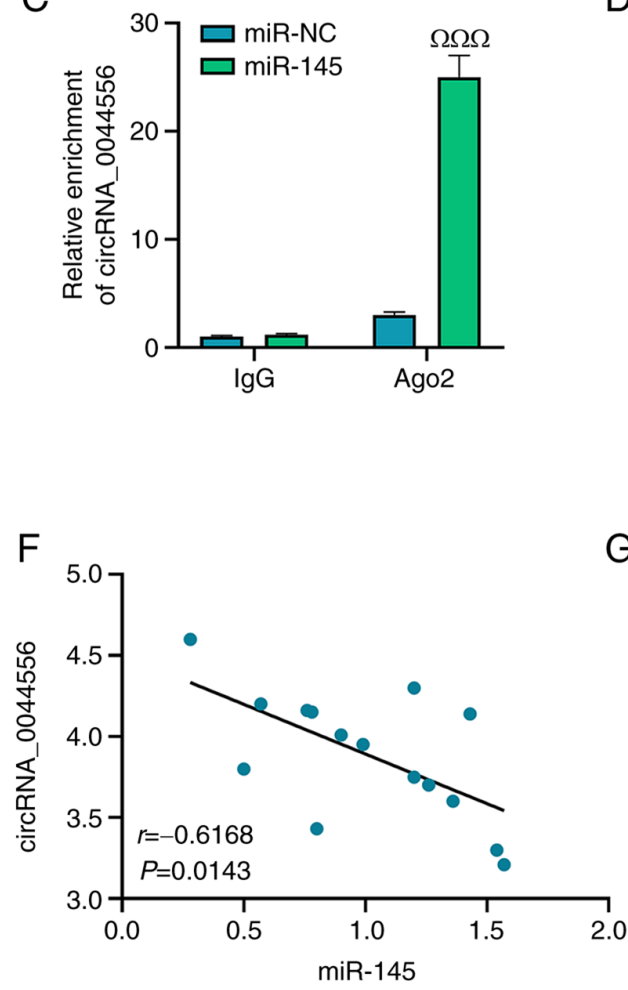

D

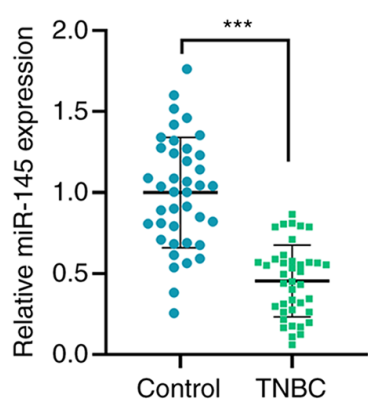

$G$

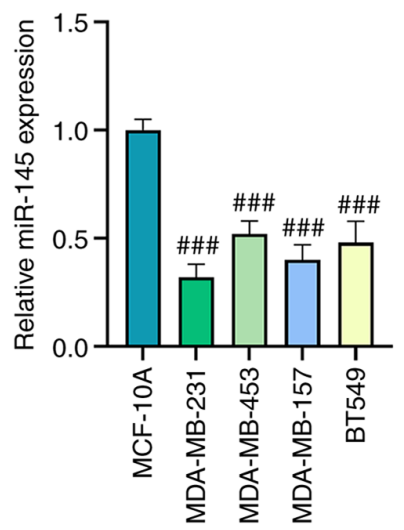

$\mathrm{B}$

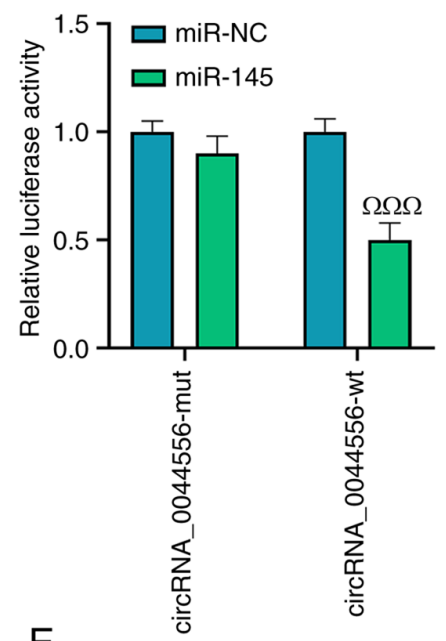

E

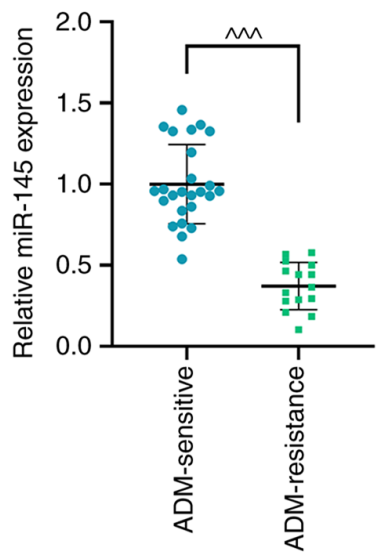

$\mathrm{H}$

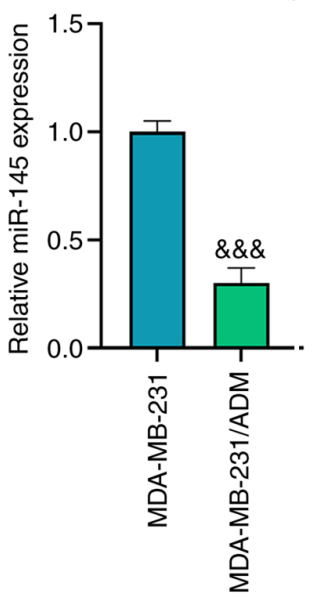

Figure 4. MiR-145 is a novel target of circRNA_0044556 and its expression is identified in TNBC tissues and TNBC cells with or without ADM treatment. (A) The circInteractome website was used to predict the binding sites between circRNA_0044556 and miR-145. (B) Subsequently, a dual luciferase reporter assay was carried out for the validation that miR-145 was sponged by circRNA_0044556. (C) An RNA binding protein immunoprecipitation assay was performed to detect the association of miR-145 and circRNA_0044556 in TNBC cells. (D and E) The expression of miR-145 in TNBC tissues with or without ADM-resistance was determined by RT-qPCR. GAPDH was used as the internal control. (F) The correlation between circRNA_0044556 and miR-145 was analyzed with Pearson's correlation analysis. (G and H) The expression of miR-145 in normal mammary epithelial cells and TNBC cells with or without ADM-resistance was determined by RT-qPCR. GAPDH was used as the internal control. ${ }^{\Omega \Omega \Omega} \mathrm{P}<0.001 \mathrm{vs}$. miR-NC; ${ }^{* * * *} \mathrm{P}<0.001 \mathrm{vs}$. Control; ${ }^{\wedge \wedge} \mathrm{P}<0.001$ vs. ADM-sensitive; ${ }^{\# \#} \mathrm{P}<0.001$ vs. MCF-10A and ${ }^{\& \& \&} \mathrm{P}<0.001$ vs. MDA-MB-231. miR, microRNA; circRNA, circular RNA; TNBC, triple-negative breast cancer; ADM, adriamycin; RT-qPCR, reverse transcription-quantitative PCR; NC, negative control.

MDA-MB-231 cells, and abrogated the effects of overexpressed circRNA_0044556 on the apoptosis and migration of parental cells (Fig. 6A and C; $\mathrm{P}<0.05$ ). In ADM-resistant MDA-MB-231 cells, the knockdown of miR-145 dampened apoptosis, facilitated migration, and reversed the effects of circRNA_0044556 silencing (Fig. 6B and D; $\mathrm{P}<0.05$ ).
MiR-145 targets NRAS, which is overexpressed in TNBC tissues and cells with or without ADM treatment. Following analysis by StarBase, NRAS was predicted to contain the binding site for miR-145 (Fig. 7A), the existence of which was then evidenced in the dual-luciferase reporter assay, where a decrease of luciferase activity in TNBC cells co-transfected 
A

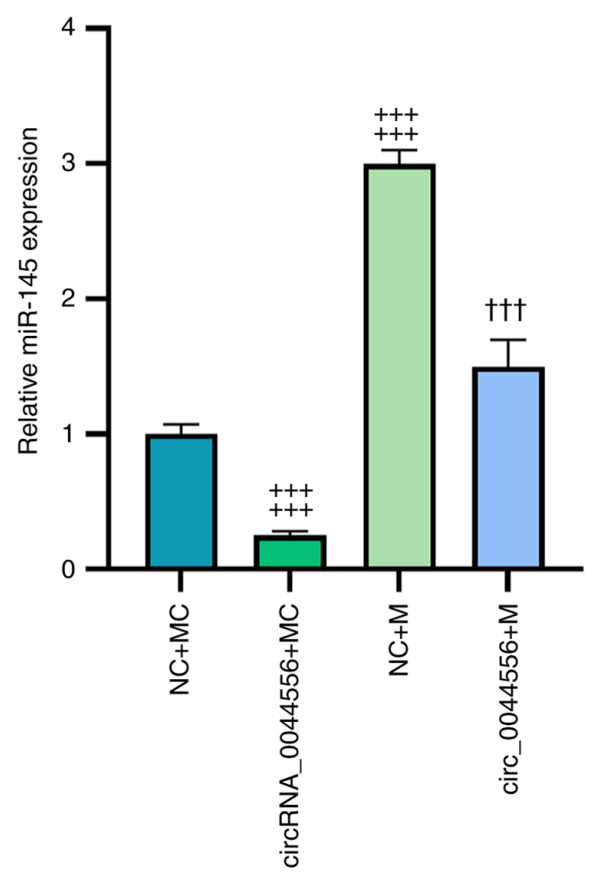

B

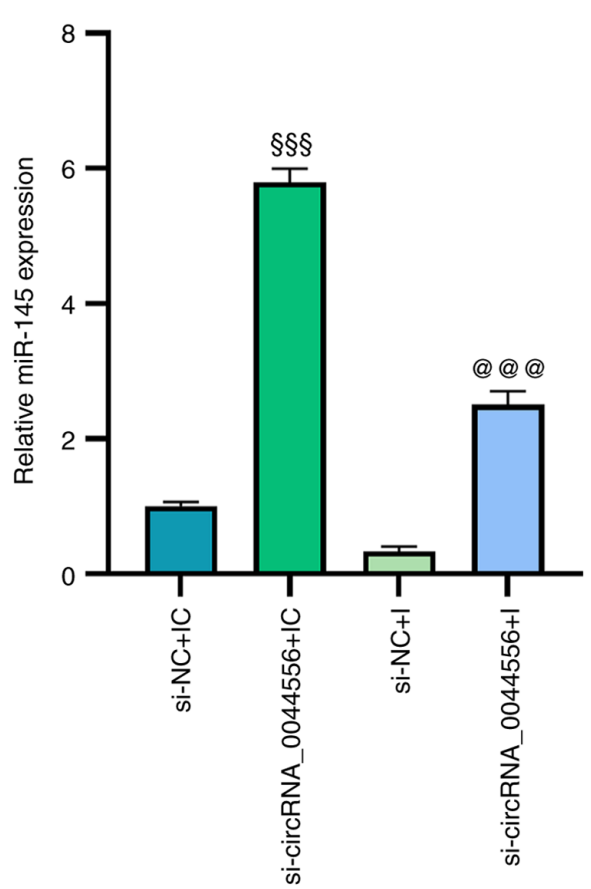

Figure 5. CircRNA_0044556 regulates the expression of miR-145 in TNBC cells with or without ADM treatment. (A and B) MDA-MB-231 cells were transfected with circRNA_0044556 overexpression plasmids, miR-145 mimic or both and MDA-MB-231/ADM cells were transfected with si-circRNA_0044556, miR-145 inhibitor or both, and the expression of miR-145 was calculated by reverse transcription-quantitative PCR. U6 was used as the internal control. ${ }^{+}+\mathrm{P}<0.001$ vs. $\mathrm{NC}+\mathrm{MC} ;{ }^{\dagger \dagger} \mathrm{P}<0.001$ vs. $\mathrm{NC}+\mathrm{M} ;{ }^{\$} \$ \mathrm{P}<0.001$ vs. si-NC+IC; and ${ }^{@ @ @ ~} \mathrm{P}<0.001$ vs. si-NC+I. NC+MC: MDA-MB-231 cells were transfected with empty plasmids (negative control for circRNA_0044556) and mimic control (MC for miR-145); circRNA_0044556+MC: MDA-MB-231 cells were transfected with circRNA_0044556 overexpression plasmids and mimic control; NC+M: MDA-MB-231 cells were transfected with empty plasmids (negative control for circRNA_0044556) and miR-145 mimic; circRNA_0044556+M: MDA-MB-231 cells were transfected with circRNA_0044556 overexpression plasmids and miR-145 mimic; si-NC+IC: MDA-MB-231/ADM cells were transfected with si-NC (negative control for si-circRNA_0044556) and inhibitor control (IC for miR-145); si-circRNA_0044556+IC: MDA-MB-231/ADM cells were transfected with si-circRNA_0044556 and inhibitor control; si-NC+I: MDA-MB-231/ADM cells were transfected with si-NC and miR-145 inhibitor; si-circRNA_0044556+I: MDA-MB-231/ADM cells were transfected with si-circRNA_0044556 and miR-145 inhibitor. circRNA, circular RNA; miR, microRNA; TNBC, triple-negative breast cancer; ADM, adriamycin; si-, small interfering; NC, negative control; I, inhibitor; M, mimic; IC, inhibitor control; MC, mimic control.

with miR-145 mimic and NRAS-wt was identified (Fig. 7B; $\mathrm{P}<0.001)$. In addition, NRAS was revealed to be overexpressed in TNBC tissues and cells with or without ADM treatment, according to the results of RT-qPCR (Fig. 7C-F; P<0.001). Furthermore, NRAS was negatively correlated with miR-145 (Fig. 7G; r=-0.6552, $\mathrm{P}=0.008$ ), and positively correlated with circRNA_0044556 in TNBC cells (Fig. 7H; r=0.6391, $\mathrm{P}=0.0103)$. circRNA_0044556 overexpression promoted the expression of NRAS in MDA-MB-231 cells, and silencing of circRNA_0044556 inhibited the expression of NRAS in ADM-resistant MDA-MB-231 cells (Fig. 7I and J; P<0.001).

\section{Discussion}

Chemotherapy is an important part of the comprehensive treatment of breast cancer (17). However, the resistance that develops in TNBC patients receiving ADM greatly limits clinical efficacy and is one of the culprits that ultimately leads to poor prognosis (18). The discovery of new chemotherapeutic drugs is a difficult and lengthy process, and as such, unravelling the mechanism of resistance to ADM in TNBC cells will facilitate the in-depth use of this original classical chemotherapeutic agent.

Recent studies have demonstrated that the development of drug resistance in cancer cells is closely linked to the aberrant expression of one or more genes and the activation of related signaling pathways $(19,20)$. The aberrancy of circRNAs is related to the sensitivity of breast cancer cells to chemotherapeutic agents. Sang et al identified that the knockdown of circRNA_0025202 in breast cancer bolsters tamoxifen resistance and contributes to tumor progression by sponging miR-182-5p (14). In addition, Liang et al revealed that circRNA_KDM4C is a potential tumor suppressor in breast cancer and markedly attenuates the resistance to ADM (21). In addition, the overexpression of circRNA_UBE2D2 in TNBC was revealed to facilitate cell proliferation and metastasis, whilst decreasing the sensitivity to doxorubicin (22). Collectively, it is suggested that circRNAs are implicated in promoting or suppressing chemosensitivity in breast cancer. CircRNA_0044556 is a newly identified circRNA that is highly expressed in colorectal cancer and positively associated to tumor stage and lymphatic metastasis (23). Intriguingly, circRNA_0044556 was aberrantly expressed in ADM-resistant TNBC. Nevertheless, its participation in the development of resistance to ADM in TNBC and its effects on the fate of TNBC cells have not been identified. In the present study, it was revealed that circRNA_0044556 was highly expressed in TNBC cells and in particular in ADM-resistant cells. In accordance with a recent study, the main role of chemotherapy drugs is to induce apoptosis in tumor cells and inhibit their growth and metastasis (24). 

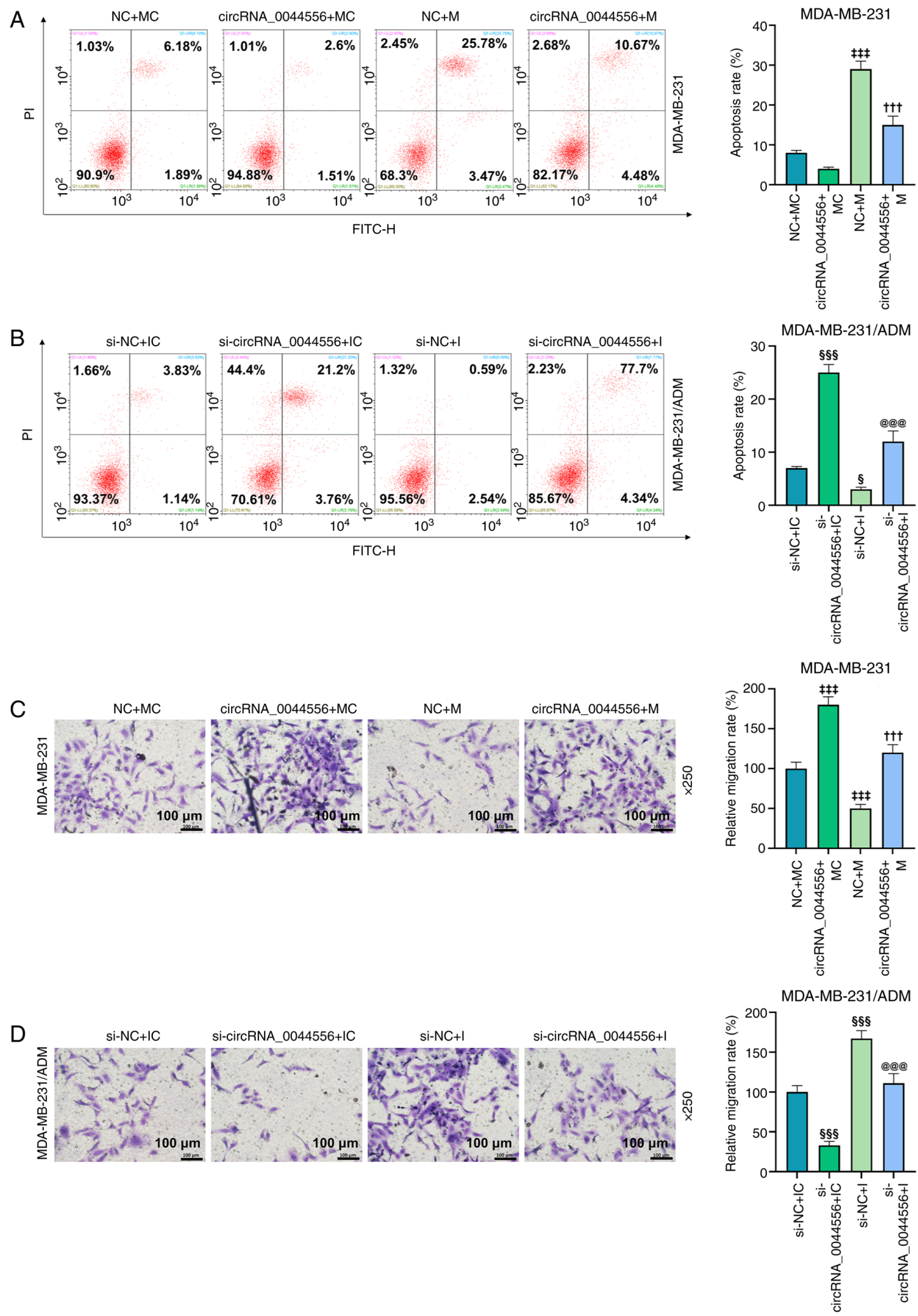

Figure 6. Regulatory role of the circRNA_0044556/miR-145 axis in the development of TNBC cells with or without ADM treatment. (A and B) Flow cytometry was employed to analyze the apoptosis after the overexpression or depletion of circRNA_0044556, miR-145 alone or in combination in MDA-MB-231 and MDA-MB-231/ADM cells. (C and D) The migratory capacities of TNBC cells after various transfections were evaluated by Transwell assay. ${ }^{\dagger+} \mathrm{P}<0.001$ vs. NC+MC; ${ }^{\dagger+} \mathrm{P}<0.001$ vs. NC+M; ${ }^{\circledR} \mathrm{P}<0.05$ and ${ }^{\$ \$ \$} \mathrm{P}<0.001$ vs. si-NC+IC; and ${ }^{\circledR} @{ }^{\circledR} \mathrm{P}<0.001$ vs. si-NC+I. NC+MC: MDA-MB-231 cells were transfected with empty plasmids (negative control for circRNA_0044556) and mimic control (MC for miR-145); circRNA_0044556+MC: MDA-MB-231 cells were transfected with circRNA_0044556 overexpression plasmids and mimic control; NC+M: MDA-MB-231 cells were transfected with empty plasmids (negative control for circRNA_0044556) and miR-145 mimic; circRNA_0044556+M: MDA-MB-231 cells were transfected with circRNA_0044556 overexpression plasmids and miR-145 mimic; si-NC+IC: MDA-MB-231/ADM cells were transfected with si-NC (negative control for si-circRNA_0044556) and inhibitor control (IC for miR-145); si-circRNA_0044556+IC: MDA-MB-231/ADM cells were transfected with si-circRNA_0044556 and inhibitor control; si-NC+I: MDA-MB-231/ADM cells were transfected with si-NC and miR-145 inhibitor; si-circRNA_0044556+I: MDA-MB-231/ADM cells were transfected with si-circRNA_0044556 and miR-145 inhibitor. circRNA, circular RNA; miR, microRNA; TNBC, triple-negative breast cancer; ADM, adriamycin; si-, small interfering; NC, negative control; I, inhibitor; M, mimic; IC, inhibitor control; MC, mimic control. 
A

$\begin{array}{cccc}\text { NRAS-wt } & \text { Target: } & 5^{\prime} \text { caaacccuunaccAUGACUGGAa } & 3^{\prime} \\ \text { miR-145 } & \text { miRNA: } & 3^{\prime} \text { ucccuaaggacccUUUUGACCUg } & 5^{\prime} \\ \text { NRAS-mut } & \text { Target: } & 5^{\prime} \text { caaacccuuaccCUGUCUCGUa } & 3^{\prime}\end{array}$

C

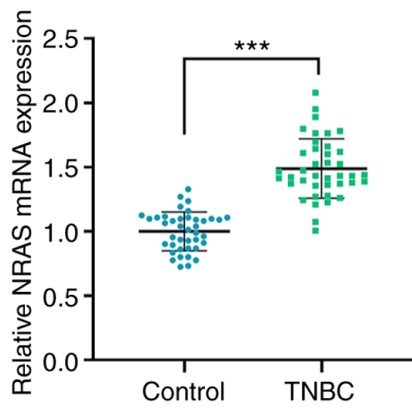

$\mathrm{D}$

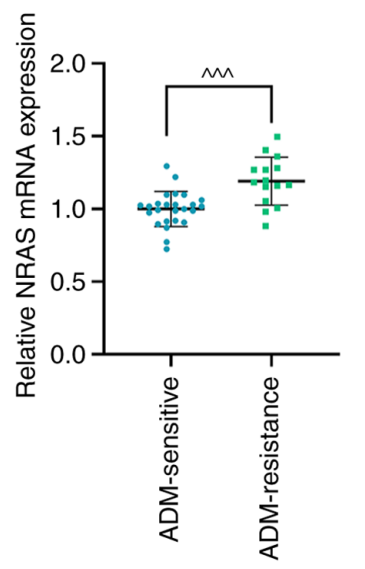

$\mathrm{B}$

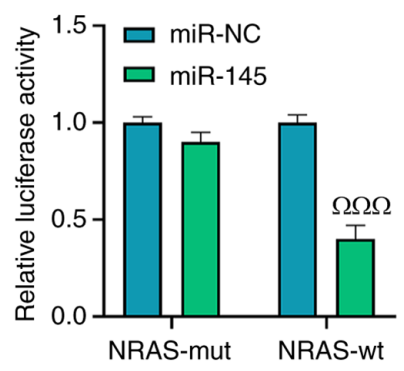

E

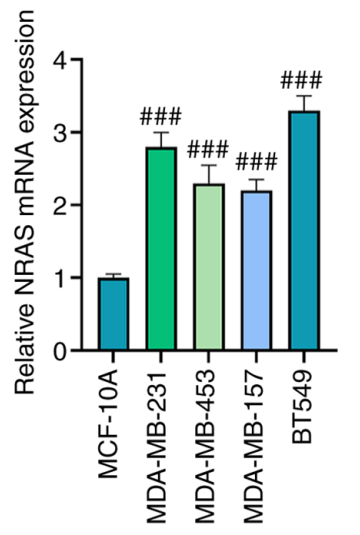

$\mathrm{F}$

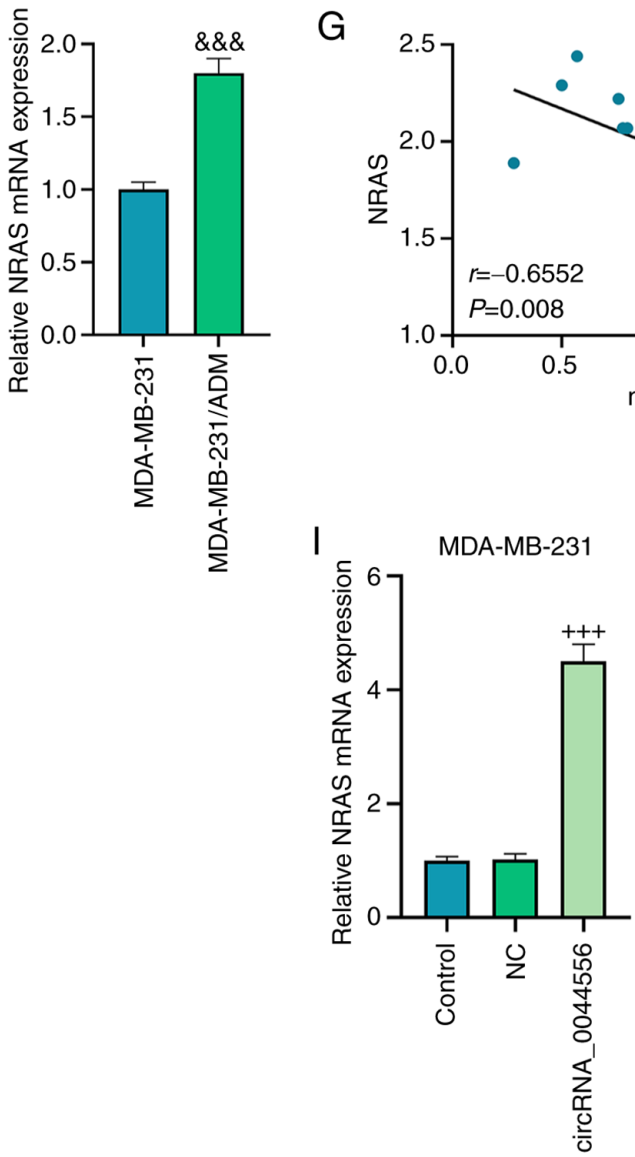

$\mathrm{H}$

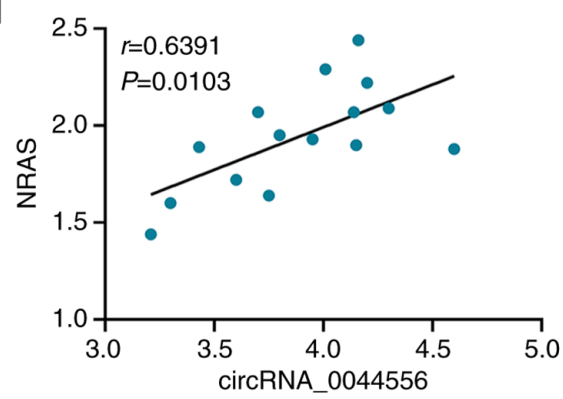

$J$

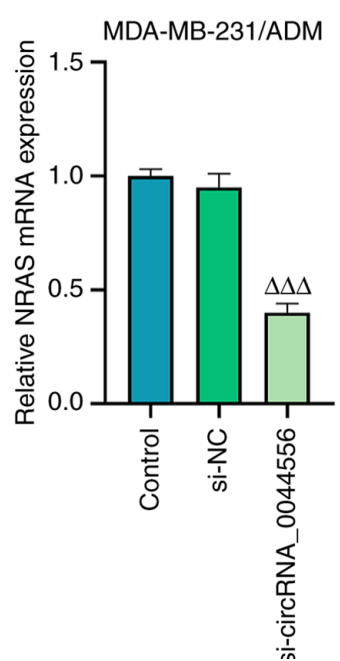

Figure 7. NRAS is targeted by miR-145 and the effect of circRNA_0044556/miR-145 axis on TNBC cells with or without ADM treatment is mediated by NRAS (A) The potential binding site of NRAS for miR-145 was predicted by StarBase website. (B) A dual luciferase reporter assay was carried out for the validation that NRAS was targeted by miR-145. (C-F) The expression of NRAS was determined in TNBC tissues, ADM-sensitive TNBC tissues, ADM-resistant TNBC tissues, normal mammary epithelial cells and cells with or without ADM-resistance by reverse transcription-quantitative PCR. GAPDH was used as the internal control. (G and H) The correlation between miR-145 and NRAS, or between circRNA_0044556 and NRAS in TNBC was analyzed with Pearson's correlation analysis. (I and J) The expression of NRAS was detected after the overexpression or silencing of circRNA_0044556 in MDA-MB-231 and MDA-MB-231/ADM cells by reverse transcription-quantitative PCR. ${ }^{\Omega \Omega \Omega} \mathrm{P}<0.001$ vs. miR-NC; ${ }^{* * *} \mathrm{P}<0.001$ vs. Control; $\wedge \wedge \wedge \mathrm{P}<0.001$ vs. ADM-sensitive; $\# \#$ $<<0.001$ vs. MCF-10A; \&\&\&P<0.001 vs. MDA-MB-231; ${ }^{++} \mathrm{P}<0.001$ vs. NC; and ${ }^{\triangle} \triangle \Delta \mathrm{P}<0.001$ vs. si-NC. NRAS, NRAS proto-oncogene, GTPase; miR, microRNA; circRNA, circular RNA; TNBC, triple-negative breast cancer; ADM, adriamycin. 
In addition, suppressing circRNA_0044556 significantly enhanced the sensitivity of TNBC cells to ADM, resulting in decreased cell viability, increased apoptosis and attenuated migration. Based on these findings, it was concluded that circRNA_0044556, which was considerably expressed at a high level in TNBC cells, could be a pivotal factor implicated in a mechanism via which TNBC cells develop the resistance to ADM, suggesting that circRNA_0044556 was indeed involved in the sensitivity of TNBC cells to ADM, which may provide new therapeutic directions to overcome this obstacle to tumor chemotherapy.

The ceRNA mechanism, as one of the biological functions of circRNAs, is creating a wave of interest in cancer research, where the circRNAs sponge miRNAs and competitively regulate the expression of downstream target genes, thus exerting their biological functions (25). Through the screening of a database and the validation of target miRNAs, it was revealed that circRNA_0044556 negatively regulated miR-145 in TNBC cells with or without ADM-resistance. Subsequent cellular functional experiments indicated that circRNA_0044556 interacted with miR-145 to affect the sensitivity of TNBC cells to ADM. The study conducted by Gao et al revealed that miR-145 mimic could enhance the sensitivity of breast cancer to the chemotherapy of ADM by targeting multidrug resistance-associated protein 1 (MRP1) (26). Ding et al demonstrated that downregulation of miR-145 promoted the proliferative and migratory capacities of breast cancer cells (27). Collectively, in our present study, the underlying mechanism of the effect of circRNA_0044556 on the ADM-resistance in TNBC was achieved by regulating miR-145 expression.

NRAS is a member of the RAS gene family and possesses the ability to bind GTP/GDP and GTPase, which controls cell growth under normal physiological conditions (28). Previous studies have revealed that NRAS is aggressively expressed in multiple cancers and its mutation could promote the progression of tumors (29-31). In our subsequent study with regard to the regulation of miR-145 on the expression of mRNA, it was identified that NRAS was targeted by miR-145 and expressed at a high level in ADM-resistant TNBC tissues and cells. Based on the study of drug resistance in breast cancer by Song et al, miRNA-22 could sensitize tumor cells to paclitaxel by targeting NRAS (32). Additionally, circ_0000073/miR-145-5p/NRAS axis has been revealed to regulate the methotrexate resistance of osteosarcoma (33). Based on our correlation analysis, it was revealed that NRAS was negatively correlated with miR-145, yet it was positively correlated with circRNA_0044556, providing evidence that the circRNA_0044556/miR-145/NRAS axis may be implicated in the development of ADM resistance in TNBC cells. However, the present study had certain limitations; rescue experiments regarding the roles of miR-145 and NRAS in ADM-resistant TNBC cells were not performed.

Overall, the present study firstly demonstrated, to the best of our knowledge, that circRNA_0044556 decreased the sensitivity of TNBC cells to ADM by sponging miR-145, which may be achieved by NRAS, indicating that circRNA_0044556 could be applied as a biomarker for diagnostic purposes or as an adjunct for the efficacy of chemotherapy using ADM in TNBC.

\section{Acknowledgements}

Not applicable.

\section{Funding}

The present study was supported by Hebei Province 2021 Medical Science Research Project Plan (grant no. 20211662).

\section{Availability of data and materials}

The datasets used and/or analyzed generated during the current study are available from the corresponding author on reasonable request.

\section{Authors' contributions}

JC and PS made substantial contributions to the conception and design of the study. JZ, YL, JM, YZ and HL performed data acquisition, data analysis and interpretation. JC and PS drafted the article or critically revised it for important intellectual content. JC and PS confirm the authenticity of all the raw data. All authors read and approved the final version of the manuscript. All authors agreed to be accountable for all aspects of the work in ensuring that questions related to the accuracy or integrity of the work are appropriately investigated and resolved.

\section{Ethics approval and consent to participate}

All procedures performed in studies involving human participants were in accordance with the ethical standards of the institutional and/or national research committee and with the 1964 Helsinki declaration and its later amendments or comparable ethical standards. The present study was approved (approval no. TNBC20190304) by the Ethics Committee of Tangshan People's Hospital (Tangshan, China).

\section{Patient consent for publication}

Not applicable.

\section{Competing interests}

The authors declare that they have no competing interests.

\section{References}

1. Zuo T, Zeng H, Li H, Liu S, Yang L, Xia C, Zheng R, Ma F, Liu L, Wang N, et al: The influence of stage at diagnosis and molecular subtype on breast cancer patient survival: A hospital-based multi-center study. Chin J Cancer 36: 84, 2017.

2. Coates AS, Winer EP, Goldhirsch A, Gelber RD, Gnant M, Piccart-Gebhart M, Thürlimann B, Senn HJ, Panel Members, André $\mathrm{F}$, et al: Tailoring therapies-improving the management of early breast cancer: St gallen international expert consensus on the primary therapy of early breast cancer 2015. Ann Oncol 26: 1533-1546, 2015.

3. Kumar P and Aggarwal R: An overview of triple-negative breast cancer. Arch Gynecol Obstet 293: 247-269, 2016.

4. Parikh RR, Yang Q, Higgins SA and Haffty BG: Outcomes in young women with breast cancer of triple-negative phenotype: The prognostic significance of CK19 expression. Int J Radiat Oncol Biol Phys 70: 35-42, 2008. 
5. Lehmann BD, Pietenpol JA and Tan AR: Triple-negative breast cancer: Molecular subtypes and new targets for therapy. Am Soc Clin Oncol Educ Book: e31-e39, 2015.

6. Gao D, Zhang X, Liu B, Meng D, Fang K, Guo Z and Li L: Screening circular RNA related to chemotherapeutic resistance in breast cancer. Epigenomics 9: 1175-1188, 2017.

7. Song J, Yin J, Bai Z, Zhang J, Meng H, Cai J, Deng W, Ma X and Zhang Z: The profile of serum microRNAs predicts prognosis for resected gastric cancer patients receiving platinum-based chemotherapy. Dig Dis Sci 62: 1223-1234, 2017.

8. Yang M, Li Y, Ruan Y, Lu Y, Lin D, Xie Y, Dong B, Dang Q and Quan C: CLDN6 enhances chemoresistance to ADM via AF-6/ERKs pathway in TNBC cell line MDAMB231. Mol Cell Biochem 443: 169-180, 2018

9. Li Z, Chen Z, Hu G and Jiang Y: Roles of circular RNA in breast cancer: Present and future. Am J Transl Res 11: 3945-3954, 2019.

10. Jahani S, Nazeri E, Majidzadeh AK, Jahani M and Esmaeili R Circular RNA; a new biomarker for breast cancer: A systematic review. J Cell Physiol 235: 5501-5510, 2020.

11. Guo JU, Agarwal V, Guo H and Bartel DP: Expanded identification and characterization of mammalian circular RNAs. Genome Biol 15: 409, 2014

12. Meng S, Zhou H, Feng Z, Xu Z, Tang Y,LiP and Wu M: CircRNA: Functions and properties of a novel potential biomarker for cancer. Mol Cancer 16: 94, 2017.

13. Shen Z, Zhou L, Zhang C and Xu J: Reduction of circular RNA Foxo3 promotes prostate cancer progression and chemoresistance to docetaxel. Cancer Lett 468: 88-101, 2020.

14. Sang Y, Chen B, Song X, Li Y, Liang Y, Han D, Zhang N, Zhang H, Liu Y, Chen T, et al: circRNA 0025202 regulates tamoxifen sensitivity and tumor progression via regulating the miR-182-5p/FOXO3a axis in breast cancer. Mol Ther 27: $1638-1652,2019$.

15. Eisenhauer EA, Therasse P, Bogaerts J, Schwartz LH, Sargent D, Ford R, Dancey J, Arbuck S, Gwyther S, Mooney M, et al: New response evaluation criteria in solid tumours: Revised RECIST guideline (version 1.1). Eur J Cancer 45: 228-247, 2009.

16. Livak KJ and Schmittgen TD: Analysis of relative gene expression data using real-time quantitative PCR and the 2(-Delta Delta C(T)) method. Methods 25: 402-408, 2001.

17. Akram $M$ and Siddiqui SA: Breast cancer management: Past, present and evolving. Indian J Cancer 49: 277-282, 2012.

18. Yin Y, Wang X, Li T, Ren Q, Li L, Sun X, Zhang B, Wang X, $\mathrm{Han} \mathrm{H}, \mathrm{He} \mathrm{Y}$, et al: MicroRNA-221 promotes breast cancer resistance to adriamycin via modulation of PTEN/Akt/mTOR signaling. Cancer Med 9: 1544-1552, 2020.

19. Vasan N, Baselga J and Hyman DM: A view on drug resistance in cancer. Nature 575: 299-309, 2019.

20. Nikolaou M, Pavlopoulou A, Georgakilas AG and Kyrodimos E: The challenge of drug resistance in cancer treatment: A current overview. Clin Exp Metastasis 35: 309-318, 2018.

21. Liang Y, Song X, Li Y, Su P, Han D, Ma T, Guo R, Chen B, Zhao W, Sang $\mathrm{Y}$, et al: circKDM4C suppresses tumor progression and attenuates doxorubicin resistance by regulating miR-548p/PBLD axis in breast cancer. Oncogene 38: 6850-6866, 2019.
22. Dou D, Ren X, Han M, Xu X, Ge X, Gu Y, Wang X and Zhao S: CircUBE2D2 (hsa_circ_0005728) promotes cell proliferation, metastasis and chemoresistance in triple-negative breast cancer by regulating miR-512-3p/CDCA3 axis. Cancer Cell Int 20: 454, 2020.

23. Jing $\mathrm{L}$, Wu J, Tang $\mathrm{X}$, Ma M, Long F, Tian B and Lin C: Identification of circular RNA hsa_circ_0044556 and its effect on the progression of colorectal cancer. Cancer Cell Int 20: 427, 2020.

24. Yamaguchi R, Lartigue L and Perkins G: Targeting Mcl-1 and other Bcl-2 family member proteins in cancer therapy. Pharmacol Therap 195: 13-20, 2019.

25. Tang YY, Zhao P, Zou TN, Duan JJ, Zhi R, Yang SY, Yang DC and Wang XL: Circular RNA hsa_circ_0001982 promotes breast cancer cell carcinogenesis through decreasing miR-143. DNA Cell Biol 36: 901-908, 2017.

26. Gao M, Miao L, Liu M, Li C, Yu C, Yan H, Yin Y, Wang Y, Qi X and Ren J: miR-145 sensitizes breast cancer to doxorubicin by targeting multidrug resistance-associated protein-1. Oncotarget 7: 59714-59726, 2016.

27. Ding Y, Zhang C, Zhang J, Zhang N, Li T, Fang J, Zhang Y, Zuo F, Tao Z, Tang S, et al: miR-145 inhibits proliferation and migration of breast cancer cells by directly or indirectly regulating TGF- $\beta 1$ expression. Int J Oncol 50: 1701-1710, 2017.

28. Chen S, Li F, Xu D, Hou K, Fang W and Li Y: The function of RAS mutation in cancer and advances in its drug research. Curr Pharm Des 25: 1105-1114, 2019.

29. Druillennec $S$, Pouponnot $C$ and Eychène A: NRAS-driven melanoma: A RAF can hide another. Mol Cell Oncol 4: e1344758, 2017.

30. Banys-Paluchowski M, Milde-Langosch K, Fehm T, Witzel I, Oliveira-Ferrer L, Schmalfeldt B and Müller V: Clinical relevance of H-RAS, K-RAS, and N-RAS mRNA expression in primary breast cancer patients. Breast Cancer Res Treat 179: 403-414, 2020

31. Prior IA, Lewis PD and Mattos C: A comprehensive survey of Ras mutations in cancer. Cancer Res 72: 2457-2467, 2012.

32. Song YK, Wang Y, Wen YY, Zhao P and Bian ZJ: MicroRNA-22 suppresses breast cancer cell growth and increases paclitaxel sensitivity by targeting NRAS. Technol Cancer Res Treat 17: 1533033818809997, 2018.

33. Li X, Liu Y, Zhang X, Shen J, Xu R, Liu Y and Yu X: Circular RNA hsa_circ_0000073 contributes to osteosarcoma cell proliferation, migration, invasion and methotrexate resistance by sponging miR-145-5p and miR-151-3p and upregulating NRAS Aging (Albany NY) 12: 14157-14173, 2020.

This work is licensed under a Creative Common Attribution-NonCommercial-NoDerivatives 4.0 International (CC BY-NC-ND 4.0) License. 